\title{
Instantons and Mirror K3 Surfaces
}

\author{
Fedor Bogomolov ${ }^{1}$ and Peter J. Braam ${ }^{2}$ \\ 1 Steklov Institute, Moscow, USSR \\ 2 University of Oxford, Oxford, England and University of Utah, Salt Lake City, USA
}

Received July 26, 1991

\begin{abstract}
The instanton moduli space of a real 4-dimensional torus is an 8-dimensional Calabi-Yau manifold. Associated to this Calabi-Yau manifold are two (singular) K3 surfaces, one a quotient, the other a submanifold of the moduli space; both carry a natural Calabi-Yau metric. They are curiously related in much the same way as special examples of complex 3-dimensional mirror manifolds; however, in our case the "mirror" is present in the form of instanton moduli.
\end{abstract}

\section{Introduction}

In the study of connections on a bundle $P \rightarrow \mathbb{T}$ over a Riemannian 4-manifold $\mathrm{T}$ the object of primary interest is the moduli space $\mathscr{M}(P)$ of anti-self-dual connections on $P$. These moduli spaces inherit various structures from $\mathbb{T}$ : when $\mathbb{T}$ is a projective algebraic variety, $\mathscr{M}(P)$ is quasiprojective, and when $\mathbb{T}$ carries a hyperkähler metric then so does $\mathscr{M}(P)$.

An important class of 4-manifolds are the flat tori, and in [BMT] it was investigated what the structure of $\mathscr{M}(P)$ is when the bundle $P$ on $T$ satisfies

$$
p_{1}(P)=-4, \quad w_{2}(P) \neq 0, \quad w_{2}(P)^{2}=0 .
$$

It was found that on $\mathscr{M}(P)$ we have a $\mathbb{T}$-action through translating connections and the quotient $\mathscr{M}=\mathscr{M}(P) / T$ admits a compactification to a Todorov surface $\overline{\mathscr{M}}=\mathscr{M} \cup \infty$ with a natural hyperKähler metric induced from the $L^{2}$ metric on the space of connections on $P$. A Todorov surface is a K3 orbifold.

The moduli space $\mathscr{M}$ is not compact because instantons can bubble off, compare [FU], and the crux of the argument is that the hyperKähler metric extends over $\infty$, as an orbifold metric. The point $\infty$ is a $D_{4}$ singularity in $\bar{M}$, and $\bar{M}$ has another 12 singularities of type $A_{2}$. The latter arise as quotient singularities for the $\mathbb{T}$-action on $\mathscr{M}(P)$.

In this paper we shall study a further Todorov surface associated to $\mathscr{M}(P)$. First of all, $\mathscr{M}(P)$ can be compactified to a complex orbifold by adding a torus $\mathbb{T}_{\infty}$ 
consisting of "ideal" instantons centered at the points parametrized by $\mathbb{T}_{\infty}$. The hyperKähler metric on $\mathscr{M}(P)$ extends to $\overline{\mathscr{M}}(P)$. On $\overline{\mathscr{M}}(P)$ one defines an Albanese map

$$
\overline{\mathscr{M}}(P) \rightarrow \mathbb{T} /(\mathbb{Z} / 2 \times \mathbb{Z} / 2) .
$$

Let $\tilde{\mathscr{M}}$ be the preimage of a regular value of the Albanese map. This surface is a submanifold of $\mathscr{M}(P)$, it is a $\mathrm{K} 3$ orbifold and again carries a natural hyperKähler metric. Also one sees that $\tilde{\mathscr{M}}$ is a cover of $\tilde{\mathscr{M}}$ ramified over the twelve $A_{2}$ singularities in $\overline{\mathscr{M}}$. On a generic fiber of $\mathscr{M}(P) \rightarrow \overline{\mathscr{M}}$ the Albanese map is a quotient by $\mathscr{K} \equiv \mathbb{Z} / 2 \times \mathbb{Z} / 2$, and in fact, $\mathscr{K}$ acts on $\mathscr{\mathscr { M }}$ with quotient $\overline{\mathscr{M}}$. The singularities in $\mathscr{\mathscr { M }}$ are just 4 copies of the $D_{4}$ singularity above $\infty \in \overline{\mathscr{M}}$, and the $A_{2}$ singularities in $\overline{\mathscr{M}}$ arise as quotient singularities for the $\mathscr{K}$-action.

In $[\mathrm{BMT}]$ it is also shown that the assignment

$$
\mathbb{T} \rightarrow \bar{M}
$$

induces an isomorphism between the Teichmüller space of flat tori $\mathbb{T}$ together with a bundle $P \rightarrow \mathbb{T}$ and the Teichmüller space of $\mathrm{K} 3$ surfaces with one $D_{4}$ singularity and 8 singularities of type $A_{2}$. This sort of theorem is referred to as a local Torelli theorem. Here we will also show that the assignment

$$
\mathbb{T} \rightarrow \tilde{M}
$$

satisfies a local Torelli theorem, with target space the space of K3 surfaces with four disjoint $D_{4}$ singularities.

In a certain sense the motivation for this work is given by the following general structure theorem for smooth Kähler orbifolds with trivial canonical class:

Theorem [Bog 1, Bog 2, Mic]. Let $M^{n}$ be a smooth Kähler orbifold with trivial first Chern class. Then a finite unramified cover of $M^{n}$ decomposes metrically as

$$
\prod V_{j} \times \prod K_{i} \times T^{m},
$$

where $V_{j}$ are simply connected with a one-dimensional space of holomorphic volume forms and no other holomorphic tensors, apart from powers of the volume forms, $K_{i}$ is a simply connected hyperKähler manifold and $T^{m}$ is a torus.

Of course, this unramified cover associated to $\overline{\mathscr{M}}(P)$ is the pullback of the fibration $\overline{\mathscr{M}}(P) \rightarrow \overline{\mathscr{M}}$ to $\widetilde{\mathscr{M}}$ under the quotient map $\widetilde{\mathscr{M}} \rightarrow \overline{\mathscr{M}}$.

When putting things together we see that the relationship between $\overline{\mathscr{M}}$ and $\tilde{M}$ is remarkably similar between pairs of Calabi-Yau 3-folds called, mirror manifolds, constructed from Fermat quintics.

\section{Construction of $\tilde{M}$}

\subsection{Review of Instanton Moduli}

The moduli space $\mathscr{M}(P)$ is a space of one-instantons. The end of this moduli space consists of localized anti-self-dual connections which are characterized by a center in $\mathbb{T}$, a small scale parameter in $\mathbb{R}_{>0}$ and a parameter in $S O(3) / Q$ describing the attachment of the localized connection to the approximately flat connection away 
from the center; here $Q$ is the quaternion group $\{ \pm 1, \pm i, \pm j, \pm k\}$. In [BMT] it is shown more globally that the end of $\mathscr{M}(P)$ is diffeomorphic to $\mathbb{T} \times\left(\mathbb{R}_{>0} \times S O(3) / Q\right)$. Topologically this can be compactified to $\overline{\mathscr{M}}(P)$ where the end is replaced by $\mathbb{T} \times \mathbb{C}^{2} / Q$. Furthermore, it was shown that for a generic flat torus the moduli space $\mathscr{M}(P)$ is smooth. In the sequel we shall restrict our attention to such generic tori.

On $\mathscr{M}(P)$ we have a $\mathbb{T}$-action through translating instantons. The quotient manifold $\mathscr{M} \equiv \mathscr{M}(P) / \mathbb{T}$ is an orbifold and also carries a hyperKähler metric. The $\mathbb{T}$-action on $\mathscr{M}(P)$ is generically free. Let $\mathbb{T}=V / \Lambda$ for a lattice $\Lambda$ in a fourdimensional vector space $V$. Define:

$$
\mathscr{K}=\Lambda^{*} / \Lambda
$$

with

$\Lambda^{*}=\left\{v \in V ; c_{1}(v, u) \in \mathbb{Z}\right.$ for any $c_{1} \in H^{2}(\mathbb{T} ; \mathbb{Z})$ with $c_{1}=w_{2}(P)(2)$ and any $\left.u \in A\right\}$.

One verifies easily that $\mathscr{K} \simeq \mathbb{Z} / 2 \times \mathbb{Z} / 2 \subset \mathbb{T}$. The stabilizers of the $\mathbb{T}$-action are all isomorphic to a $\mathbb{Z} / 2$ subgroup of $\mathscr{K}$, and each nonzero element of $\mathscr{K}$ has exactly 4 fixed tori in $\mathscr{M}(P)$. This results in the 12 singularities of type $A_{2}$ in $\mathscr{M}$. For background see [BMT].

As mentioned in the introduction the hyperKähler metric on $\mathscr{M}$ extends to a hyperKähler metric on $\overline{\mathscr{M}}=\mathscr{M} \cup \infty$, and $\infty$ appears as a $D_{4}$ singularity of $\overline{\mathscr{M}}$. This implies immediately that $\overline{\mathscr{M}}$ is a K3 orbifold: it has trivial canonical bundle and contains (singular) lines. For future reference we mention the obvious fact: $H^{2}(\overline{\mathscr{M}} ; \mathbb{R})=\mathbb{R}^{6}$.

\subsection{Construction of $\tilde{\mathscr{M}}$}

The $L^{2}$-metric on the space of connections induces a hyperKähler metric on $\mathscr{M}(P)$. The methods of [BMT, Sect. 2.6] carry over without change to show that:

Theorem 2.1. The hyperKähler structure on $\mathscr{M}(P)$ extends to an orbifold hyperKähler structure on $\bar{M}(P)$. The $\mathbb{T}$-action by translating instantons extends to a hyperKähler $\mathbb{T}$-action on $\overline{\mathscr{M}}(P)$.

A direct consequence of the extension of the hyperKähler metric is

Corollary 2.2. $\kappa(\overline{\mathscr{M}}(P)) \equiv \wedge_{\mathbb{C}}^{4} T^{*}(\overline{\mathscr{M}}(P))$ is holomorphically trivial, in particular, the first Chern class $c_{1}(\mathscr{M}(P))=0$.

The use of Theorem 2.1 to establish this corollary is not necessary.

On a compact Kähler manifold with trivial canonical class Serre duality $H^{0}\left(\Omega^{P}\right)$ $=H^{n}\left(\Omega^{n-p}\right)^{*}$ takes a more concrete form. It states that the pairing:

$$
H^{0}\left(\Omega^{p}\right) \otimes H^{0}\left(\Omega^{n-p}\right) \rightarrow H^{0}\left(\Omega^{n}\right) \simeq \mathbb{C}
$$

is non-degenerate. In particular, there is a duality between $H^{0}\left(\Omega^{1}\right)$ and $H^{0}\left(\Omega^{n-1}\right)$ $=H^{0}\left(T^{1,0} M\right)$, and therefore, the Albanese map

$$
M^{n} \rightarrow A\left(M^{n}\right) \equiv H^{1}\left(M^{n} ; \mathbb{Z}\right) / H^{1}\left(M^{n} ; \mathbb{C}\right) / H^{1,0}\left(M^{n}\right)
$$

given by integrating holomorphic one forms is a locally trivial fibration. We now apply this to $M^{n}=\bar{M}(P)$. 
Definition 2.3. The surface $\tilde{\mathscr{M}}$ is the fibre of the Albanese map.

The metrical properties of $\widetilde{\mathscr{M}}$ are summarized by

Proposition 2.4. The metric of $\tilde{\mathscr{M}} \subset \overline{\mathscr{M}}(P)$ is hyperKähler. The natural map $\tilde{\mathscr{M}} \rightarrow \overline{\mathscr{M}}$ is isometric away from the singular points.

Proof. This follows most easily through some symplectic considerations, compare [HKLR]. Let $S^{1} \subset T$ be a one parameter subgroup. This induces a symplectic vectorfield $X$ on $\bar{M}(P)$ and so do $I X, J X, K X$. The Albanese map is constructed by picking a base point $b$ in $\bar{M}(P)$ and integrating the one forms $i_{X} \omega, i_{I X} \omega, i_{J X} \omega, i_{K X} \omega$ along a curve from $b$ to $x \in \bar{M}(P)$, to obtain a vector in $\mathbb{R}^{4}$. The answer depends only on $x$ and the homotopy class of the path. Changing the homotopy class shifts the outcome of the integration by an element of a lattice $\Lambda^{*} \subset \mathbb{R}^{4}$.

But this is almost the definition of the hyperKähler moment map, and we see that the fibre of the Albanese map equals a ramified cover of the hyperKähler quotient of $\bar{M}(P)$ by $S^{1}$. For that reason it is hyperKähler and a ramified cover of $\overline{\mathscr{M}}$.

The proposition immediately establishes that $\tilde{\mathscr{M}}$ is a singular K3 surface, as it contains lines and has a trivial canonical bundle.

Proposition 2.5. The cover $\tilde{M} \rightarrow \bar{M}$ has degree 4 away from points of ramification. There is a subgroup $\mathscr{K} \subset \mathbb{T}$ leaving $\tilde{M}$ invariant. An unramified $\mathscr{K}$ cover of $\bar{M}(P)$ decomposes as $\tilde{\mathscr{M}} \times \mathbb{T}$.

Proof. The map $\tilde{\mathscr{M}} \rightarrow \overline{\mathscr{M}}$ is doubly ramified over exactly 12 points. The surface $\tilde{\mathscr{M}}$ has $d$ singularities of type $D_{4}$ and is smooth elsewhere, where $d$ is the covering degree. The usual formula for the Euler characteristic, or the formula for the curvature of orbifolds [KT], gives $d=4$. On all but 12 fibres the Albanese map is a 4 -fold covering, and one easily sees that $\overline{\mathscr{M}}$ is $\mathscr{K}$-invariant.

Clearly, we have that $\overline{\mathscr{M}}(P)=\widetilde{\mathscr{M}} \times_{\mathscr{K}} \mathbb{T}$.

\subsection{Local Torelli}

The Kähler structure on $\tilde{\mathscr{M}}$ will depend on that of $\mathbb{T}$, and the assignment $\mathbb{T} \rightarrow \mathscr{M}$ defines a map of the moduli space of Kähler structures on a torus to the moduli space of orbifold $\mathrm{K} 3$ surfaces with $4 \mathrm{D}_{4}$ singularities. The Teichmüller spaces of both of these are equal to

$$
S O(3,3) / S O(2) \times S O(3) \text {. }
$$

These matters are discussed in detail in the paper by Todorov [T] and Kobayashi and Todorov [KT]. Essentially the theory says that a hyperKähler structure on a torus or a $\mathrm{K} 3$ surface is determined by the real 3-dimensional subspace of self-dual forms in $H^{2}$; to avoid ambiguities one starts by fixing the 2 nd homology. A Kähler structure is then determined by further fixing a line inside this three-dimensional subspace. In the case of orbifold singularities, such as $4 D_{4}$ singularities, one fixes sixteen elements

$$
a_{j}, b_{i j} \in H^{2}(\mathrm{~K} 3 ; \mathbb{Z})=\mathbb{Z}^{22}, \quad j=1, \ldots, 4, \quad i=1,2,3
$$


which form the configuration of four disjoint $D_{4}$-singularities, that is:

$$
\begin{gathered}
\left\langle a_{k}, a_{l}\right\rangle=0 \quad \text { if } \quad k \neq l, \quad\left\langle a_{k}, a_{k}\right\rangle=-2, \\
\left\langle b_{k l}, b_{m n}\right\rangle=0 \quad \text { if } \quad(k, l) \neq(m, n), \quad\left\langle b_{k l}, b_{k l}\right\rangle=-2, \\
\left\langle a_{l}, b_{k l}\right\rangle=1, \quad\left\langle a_{l}, b_{k m}\right\rangle=0 \text { for } m \neq l .
\end{gathered}
$$

Here $\langle$,$\rangle is the intersection pairing. The space of self-dual forms on an orbifold$ K3-surface where all these lines are collapsed to 4 singular points now must have that all self-dual forms are $\langle$,$\rangle orthogonal to the span of these 16$ classes. This once again reduces us the flag manifold

$$
S O(3,3) / S O(2) \times S O(3),
$$

as the Teichmüller space.

Once the torus $\mathbb{T}$ has been fixed the moduli spaces under consideration are all submanifolds of $\mathscr{B}$ the space of irreducible connections modulo gauge transformations. There is a natural map:

$$
\mu: H^{2}(\mathbb{T}) \rightarrow H^{2}(\mathscr{B})
$$

which has the property that it maps the cohomology classes of self-dual forms on $\mathbb{T}$ to the cohomology classes of self-dual forms on $\mathscr{M}(P)$. In [BMT] it was shown that this implies without difficulty that the map $\mathbb{T} \rightarrow \overline{\mathscr{M}}$ is an isomorphism on Teichmüller space. The same holds for $\mathbb{T} \rightarrow \mathscr{M}$.

Interestingly, it appears that any K3 surface with a configuration of 4 disjoint $D_{4}$ singularities admits a $\mathscr{K}$-action.

\subsection{Mirror Manifolds}

Let $r(\overline{\mathscr{M}})$ and $r(\tilde{\mathscr{M}})$ be our K3-orbifolds with the $D_{4}$-singularities resolved in a minimal way. The foregoing discussion details the relationship between these two surfaces. It turns out that this relationship is strikingly similar to that between pairs of mirror manifolds, pairs of Calabi-Yau 3-folds constructed by physicists. For details see [Can, M].

Candelas starts with a Fermat quintic $\widetilde{X}$ and considers a $\mathscr{L} \equiv \mathbb{Z} / 5 \times \mathbb{Z} / 5 \times \mathbb{Z} / 5$ action on $\widetilde{\mathscr{X}}$. This action is free exactly on an open subvariety of Euler characteristic 0 . The quotient $\overline{\mathscr{X}}=\widetilde{\mathscr{X}} / \mathscr{L}$ has trivial canonical bundle and is a 3-dimensional Calabi-Yau orbifold. The Teichmüller spaces of pairs of holomorphic structure and Calabi-Yau metric for the spaces $\mathscr{X}$ and $\overline{\mathscr{X}}$ are isomorphic. However, infinitesimally the isomorphism between these Teichmüller spaces maps deformations of complex structure on $\widetilde{\mathscr{X}}$ to deformations of Kähler metric on $\overline{\mathscr{X}}$ and vice versa; this is summarized by a natural isomorphism $H^{11}(\widetilde{\mathscr{X}})=H^{21}(\overline{\mathscr{X}})$.

This is much the same relation as we have between $r(\tilde{M})$ and $r(\tilde{M})$. The Euler characteristic of $r(\tilde{M})$ minus the singular points for the $\mathscr{K}$-action is 0 , and again we have the equivalence of the Teichmüller spaces of pairs of holomorphic structures and Kähler metrics.

Many other examples of mirror manifolds have been found and most of them start with a Calabi-Yau 3-fold $\widetilde{\mathscr{X}}$ with a large symmetry group. One considers a 
subgroup $\mathscr{L}$ which preserves the holomorphic volume form. It happens that one can find such groups with the following properties:

1. the quotient space has a natural smooth desingularization $\bar{X}$,

2. the Euler characteristic of the subset of $\tilde{\mathscr{X}}$ where $\mathscr{L}$ acts freely is 0 ,

3 . the Euler characteristic of $\tilde{\mathscr{X}}$ is minus that of $\overline{\mathscr{X}}$.

These properties seem to force the mirror symmetry. It is not known if $\mathscr{L}$ is always abelian.

It seems interesting to explore the following

Question. Can we find examples of a Kähler torus T of some dimension and a moduli space $\overline{\mathscr{X}}(P)$ of holomorphic structures on a bundle $P \rightarrow T$, such that

1. $\mathscr{L} \subset T$ is a stabilizer group of the translation action on $\overline{\mathscr{X}}(P)$, and an Albanese map $\overline{\mathscr{X}}(P) \rightarrow T / \mathscr{L}$

2. $\overline{\mathscr{X}}=\overline{\mathscr{X}}(P) /$ T and a preimage $\tilde{\mathscr{X}}$ of the Albanese map form a pair of mirror CalabiYau threefolds.

This would construct the mirror manifolds and the moduli functor would be the mirror relating the two.

The key point of interest in the study of mirror manifolds are special functions defined on the Teichmuller spaces which encode the number of lines in the mirror 3-folds through their Taylor expansions. A question of farther reach would be if these functions could be explored through the use of bundles.

\section{References}

[Bog 1] Bogomolov, F.: Kähler manifolds with trivial canonical class. Izvestiya Akad. Nauk. SSSR Ser. Mat. 38, 11-21 (1974)

[Bog 2] Bogomolov, F.: The decomposition of Kähler manifolds with trivial canonical class. Mat. Sb. (N.S.) 93 (135), 573-575 (1974)

[BMT] Braam, P.J., Maciocia, A., Todorov, A.: Instanton moduli as a novel map from Tori to K3 surfaces. Preprint (1991)

[Can] Candelas, P., Ossa, X. de la, Green, P., Parkes, L.: A pair of Calabi-Yau manifolds as an exactly soluble super conformal theory. Nucl. Phys. B 359, 21-74 (1991)

[FU] Freed, D.S., Uhlenbeck, K.K.: Instantons and four-manifolds. Berlin, Heidelberg, New York: Springer 1984

[HKLR] Hitchin, N., Karlhede, A., Lindstrom, U., Rocek, M.: HyperKähler metrics and super symmetry. Commun. Math. Phys. 108, 535-589 (1987)

[KT] Kobayashi, R., Todorov, A.: Polarized period map for generalized K3 surfaces and the moduli of Einstein metrics. Tohoku Math. J. 39, 341-363 (1987)

[M] Morrison, D.: Mirror symmetry and rational curves or quintic 3-folds: a guide for mathematicians. Duke Preprint, July 1991

[Mic] Michelsohn, M.L.: Kähler manifolds with vanishing first Chern class. Ann. Math. Studies, Princeton (1982), 359-361, Seminar on Differential Geometry

[T] Todorov, A.: Application of the Kähler-Einstein-Calabi-Yau metric to the moduli of K3 surfaces. Inv. Math. 61, 251-265 (1980) 